\title{
Application of a Reconfiguration Methodology for Multiple Assembly System Reconfigurations
}

\author{
D. Smale and S. Ratchev \\ Precision Manufacturing Centre, Faculty of Engineering, University of Nottingham, \\ Nottingham, UK
}

\begin{abstract}
Reconfigurable Assembly Systems (RAS) offer the potential to enable rapid exchange of functional modules. There has however been little investigation into the planning of multiple system reconfigurations. The work proposes a capability-based approach; consisting of a Reconfiguration Methodology, supported by a Capability Model and Taxonomy. The Methodology focuses on multiple system reconfigurations and is based upon operator-oriented definition, thereby utilising existing knowledge and expertise. The Capability Model consists of Capability Identification and Comparison processes: by aggregating the results, capability and compatibility sets can be derived. Further, the Model has strong links to the Capability Taxonomy. The work describes the overall approach and key elements and provides a detailed example application in software using a simple multi-product test case. Key conclusions are drawn and future work outlined.
\end{abstract}

Keywords: Reconfigurable Assembly, System Planning, Capabilities.

\section{Introduction}

Reconfigurable Assembly Systems (RAS) offer the ability to rapidly exchange process equipment modules, which facilitates a change in product, system and/or to provide equipment redundancy. There have been efforts in developing platforms that can be physically reconfigured [1] however; there has been little investigation into the planning of multiple system reconfigurations.

The current state-of-the-art in manufacturing systems dictates that the vast majority of new lines are bespoke and single purpose. Generally, the system is designed to be as cost effective as possible [2]. RASs are researched as a means of addressing the key issues presented by conventional systems [3]. The reconfigurability is typically achieved through the implementation of standardised mechanical, electrical and control interfaces. RASs are widely accepted as the route for future production systems [4]. There has been some effort towards identification of requirements for new or reconfigured assembly systems [5], the design of new assembly systems [3] and operation allocation [6]. However, a methodology which considers multiple system reconfigurations, and which is applicable both to new and to existing systems, is not available. 


\section{Methodology Overview}

In order to achieve the full potential offered by RAS, a new methodology must be developed. It is proposed that this includes three key elements: 1) Capability taxonomy and definition process, 2) Capability modeling and comparison and 3) Reconfiguration identification, optimization and validation methods. These elements can be further enhanced by focusing on precision production, micro-manufacturing and assembly rather than machining. The proposed approach is intended to broadly follow the, currently human-centred, complex decision-making approach from the specification of requirements by the customer through to the specification and planning of the assembly system solution. The proposed approach sequentially follows four key process elements: Capability Modeling, Capability Identification, Capability Comparison and Configuration and Sequence Analysis.

\subsection{Capability Modeling}

The modeling of the Capabilities fundamentally requires that there is a clear definition for "Capability". This definition must be applicable to both the products required and the available equipment. Furthermore, enabling a tiered or hierarchical definition with several levels of detail is of substantial benefit as this enables the application of the model to various stages in product development. This definition will constitute a Capability Taxonomy.

The proposed Capability Taxonomy is described in detail in earlier work [7]. It comprises 6 Capability Classes: Motion, Join, Retain, Measure, Feed and Work.

In addition to the clear Taxonomy, it is essential that the requirements for the project are defined. This in turn requires a clear structure of the project and the major stakeholders: a factor which is made more important when considering specification of the system early in the product development cycle. These matters are researched and solutions defined in previous work [8].

\subsection{Capability Identification}

The next stage is to identify each capability; both Required and Available. The identification of the Available Capabilities will be supported by guidelines associated with the Taxonomy. These guidelines provide a series of Yes/No questions which enable the capabilities provided by the equipment to be identified.

In order to identify the capabilities that are required by each product in order to produce them, a more structured method is required. This is termed the Process Flow Template (PFT) and is based upon the principle that assembly requires two parts to be brought and maintained together. Therefore, the core assembly processes are "Moving Part $x$ " and "Joining Parts $x$ and $y$ ". By using this as a basis for the definition of the required capabilities, a number of rules can be applied. The application of these rules to the Process Flow Template diagram produces a Capability Flow Diagram (CFD). This diagram identifies the required capabilities for one product: therefore, one diagram is needed for each product. The Capability Taxonomy is then applied in order to define each individual capability. The full details of the identification process are described in previous work [7]. 


\subsection{Capability Comparison}

The next step is to perform a comparison of the capability sets. For this purpose a Comparison Matrix is used. This concept is developed and an example provided in earlier work [7]. The matrix has been devised in order to enable two or more capability sets to be compared. Existing capabilities are listed in the first column, whilst required capabilities are listed across the first row. At each intersection a comparison is performed. Then, each row and column is totalled in order to provide the basis for configuration analysis. The result of the comparison will be one of three values: ' 1 ' meaning that the capabilities match, ' 0.1 ' meaning the capabilities are compatible but not exactly matching and ' 0 ' meaning that there is no realisable commonality between the capabilities. The ' 0.1 ' value can be viewed as the 'grey area' of the analysis and is used to represent cases where the existing capability is over-specified with respect to the required capability. Whether or not the existing capability is used or replaced is a decision based more upon the requirements of the system as a whole rather than purely on the technical matters.

\subsection{Configuration and Production Sequence Analysis}

The next stage of the methodology is to analyse the comparison results to determine the optimum configuration for each product. This analysis will focus on the previous determined 'grey area' of capabilities: those with a 0.1 value in their column of the Comparison Matrix. The decisions made and the methodology which supports them, has been described and demonstrated in earlier work [9].

At this point in the analysis, each product has an optimized solution configuration. However, there are still a number of decisions to be made and issues to be resolved, specifically finding the Production Sequence. This is the order in which each of the products is produced and is one of the most critical decisions affecting the performance and efficiency of the system. It is generally preferable to minimize the system disruption, downtime and reconfiguration effort (module exchange), thereby minimizing the overall cost of reconfiguration. This is achieved through adopting a 'productcentered' approach and identifying the commonalities between each of the products. The Production Sequencing Method is detailed below:

A new matrix is used to provide this analysis; the correlation matrix enables comparison between the Required Capabilities for the different products. As with the previous comparison process, a ' 1 ' is entered if the Capabilities are compatible, ' 0 ' is entered for non-matches. The details of the exact calculation of the Product Correlation Ratio (PCR) is shown and demonstrated in [9].

\section{Example Application}

To demonstrate the process, a highly simplified example is used. The example used is based on real equipment and processes, but considers only a very short list so that it is easy to follow and concise for the purpose of this research. One of the key innovations of this research is the consideration of multiple reconfigurations thus the example contains five products but each with only a few processes for conciseness. 
Define the Existing System Capabilities. The example existing system consists of: one SCARA type robot, one mechanical gripper, one tray feeder and a static fixture.

At this stage the Capability Taxonomy and the associated guidelines are used to define the capabilities for the existing equipment. Most equipment modules have only one capability; more flexible modules can have multiple capabilities which makes the definition more complex but in this example, each module has one capability:

- Robot: Motion $1,1,39,2,2,3$

- Gripper: Retain 3,1,2,2,

- Tray: Feed 2,3,2,2,

- Fixture: Retain 3,2,2,2,

Define the Required Capabilities. The products used for this example are:

- Product A: Cap onto a Cylinder.

- Product B: Chip on a PCB.

- Product C: Pin on a Plate.

- Product D: Sphere onto a Shaft.

- Product E: Cube into a Slot.

Using the PFT five CFDs are produced, one for each product. This enables the identification of all of the possible Required Capabilities for each product. An example of this, for Product B, is shown in Fig. 1. The five capability sets can be listed and defined and are shown in Table 1.

\section{PRODUCT B}

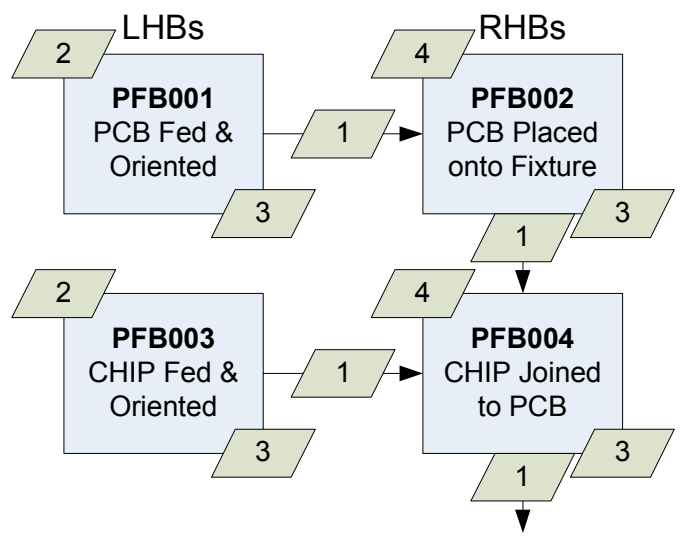

Fig. 1. The completed CapID Diagrams for the 5 products

Perform Capability Comparison. The first task is to sequence the capabilities. This is important for the selection and optimisation procedures later. Additionally, the capabilities are given a short alpha-numeric designator. Even a very simplified example of 5 2-part products results in a total of 55 required capabilities; for this reason only those capabilities for Product B (along with their definition from the Taxonomy) 
are shown in Table 2 below. This clarifies the need for the process to be automated within software. The sequenced capabilities can then be entered into the Capability Matrix. The first stage of analysis investigates each configuration separately. Each Required Capability Set is compared to the Existing Capability Set with the result being entered into the intersecting location in the matrix. The Capability Matrix for Product B is illustrated in Table 3.

Table 1. List of all of the capability sets by product

\begin{tabular}{|l|l|l|l|l|}
\hline Product A & Product B & Product C & Product D & Product E \\
\hline PFA01 & PFB01 & PFC01 & PFD01 & PFE01 \\
\hline PFA01 & PFB01 & PFC01 & PFD01 & PFE01 \\
\hline PFA01-PFA02 & PFB01-PFB02 & PFC01-PFC02 & PFD01-PFD02 & PFE01-PFE02 \\
\hline PFA02 & PFB02 & PFC02 & PFD02 & PFE02 \\
\hline PFA02 & PFB02 & PFC02 & PFD02 & PFE02 \\
\hline PFA02-PFA04 & PFB02-PFB04 & PFC02-PFC04 & PFD02-PFD04 & PFE02-PFE04 \\
\hline PFA03 & PFB03 & PFC03 & PFD03 & PFE03 \\
\hline PFA03 & PFB03 & PFC03 & PFD03 & PFE03 \\
\hline PFA03-PFA04 & PFB03-PFB04 & PFC03-PFC04 & PFD03 PFD04 & PFE03-PFE04 \\
\hline PFA04 & PFB04 & PFC04 & PFD04 & PFE04 \\
\hline PFA04 & PFB04 & PFC04 & PFD04 & PFE04 \\
\hline PFA04-OUT & PFB04-OUT & PFC04-OUT & PFD04-OUT & PFE04-OUT \\
\hline
\end{tabular}

Table 2. The full definition of the capabilities for Product B

\begin{tabular}{|l|l|l|}
\hline Cap. Locator & Cap. Designator & Cap. Definition \\
\hline PFB01-PFB02 & PB01 & $1,1,39,2,2$ \\
\hline PFB03-PFB04 & PB02 & $1,1,39,2,2$ \\
\hline PFB02-PFB04 & PB03 & $1,2,-$ \\
\hline PFB04-OUT & PB04 & $1,2,-$ \\
\hline PFB01 & PB05 & $2,2,1,1,1$ \\
\hline PFB03 & PB06 & $2,2,2,1,1$ \\
\hline PFB01 & PB07 & $3,1,1,1,1$ \\
\hline PFB03 & PB08 & $3,1,2,1,1$ \\
\hline PFB02 & PB09 & $3,2,1,1,1$ \\
\hline PFB02 & PB10 & $4,1,2,2,1,4$ \\
\hline PFB04 & PB11 & $4,1,2,2,5,4$ \\
\hline
\end{tabular}

From the results of the five comparison matrices for products a to $\mathrm{E}$, the following conclusions can be drawn with respect to the original equipment capabilities:

- The existing robot can be used in production of Products A, B, C and E

- The existing Tray Feeder cannot be used in production of any products

- The existing Mechanical Gripper can be used in production of Product B and $\mathrm{E}$ and possibly in the production of Products $\mathrm{B}$ and $\mathrm{C}$ 
Table 3. The Capability Matrix for Product B

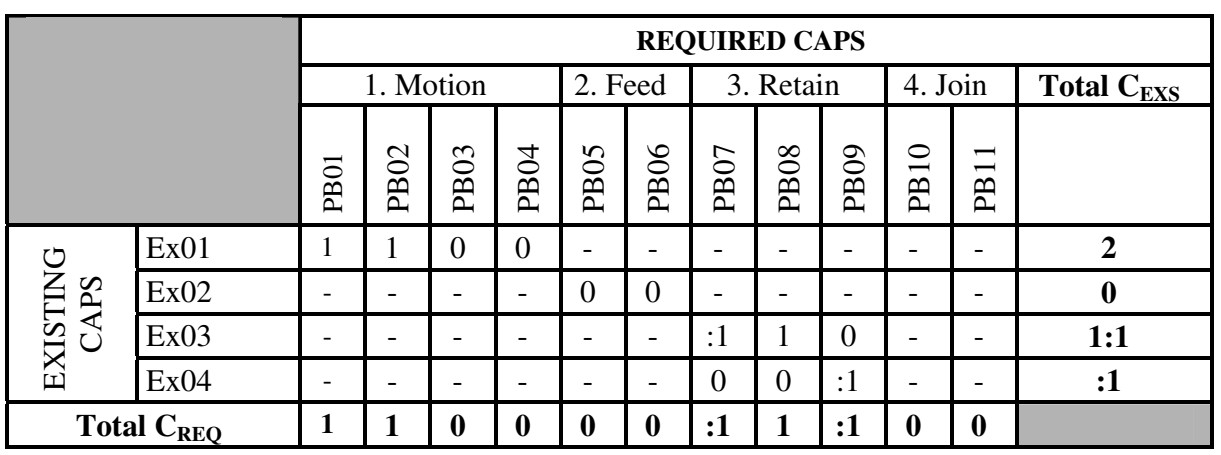

- The existing Fixture can be used I the production of Product E and possibly in the production of Products B and C

Perform Configuration Analysis. The first element of the configuration analysis is to analyse the configuration for each product independently. The overall analysis of the complete system, including equipment allocation, is the role of the Reconfiguration Methodology. At this stage of analysis, the capabilities are divided into four lists: Retained, Redundant, Investigation and Procurement. A summary of this evaluation for Product B is illustrated in Table 4.

- Retained Capabilities are the existing capabilities with a value total of 1 or more. These capabilities should be re-used in the new configurations.

- Redundant Capabilities are the existing capabilities with a value total of 0 . These capabilities will be removed and not used in the new configurations.

- Investigation Capabilities are the existing capabilities with a value total of :1 or more. These could be used in the new configurations, but their applicability will depend upon further analysis which will be conducted in the Reconfiguration Methodology.

- Procurement Capabilities are the Required Capabilities that are not met by any of the existing capabilities. These must be procured.

Table 4. A summary of the capability evaluation for Product B

\begin{tabular}{|l|l|}
\hline \multicolumn{2}{|c|}{ Product B } \\
\hline Retained Capabilities & Ex01, Ex03 \\
\hline Redundant Capabilities & Ex02 \\
\hline Investigation Capabilities & Ex03, Ex04 \\
\hline Procurement Capabilities & PB03-PB06, PB10, PB11 \\
\hline
\end{tabular}

Perform Sequence Analysis. The first element of the sequence analysis is to construct one amalgamated comparison matrix. All of the five capability sets are listed in a single matrix, this matrix is then extended vertically with a triangular "House of 
Quality" style inter-relationship grid. This is used to perform the same kind of comparison of capabilities as performed previously but between the different required sets. With each capability compared, a ratio is derived to determine the similarity between the requirements (and hence the likely similarity between the resulting configurations). This is termed the Similarity Coefficient and is defined in the form:

\section{Similarity Coefficient}

\{No. of matching capabilities\}

$$
=\frac{\text { \{No. of possible matching capabilities\} }}{\text { \{ }}
$$

In this case, the matching capabilities are those with the exact match. The number of possible matching capabilities is defined as the number where a comparison is made (different Classes are not compared and so not included in the total). The full matrix is not shown due to its size. Table 5 shows the similarity comparison results.

Table 5. The Similarity Coefficients for all of the five products in the example.

\begin{tabular}{|c|c|c|c|c|c|}
\hline & PROD A & PROD B & PROD C & PROD D & PROD E \\
\hline PROD A & N/A & $6 / 33$ & $7 / 33$ & $8 / 33$ & $5 / 33$ \\
\hline PROD B & Repeat & N/A & $4 / 33$ & $5 / 33$ & $17 / 33$ \\
\hline PROD C & Repeat & Repeat & N/A & $2 / 33$ & $13 / 33$ \\
\hline PROD D & Repeat & Repeat & Repeat & N/A & $6 / 33$ \\
\hline PROD E & Repeat & Repeat & Repeat & Repeat & N/A \\
\hline
\end{tabular}

This table enables the optimal production sequence to be indentified. This sequence is the one in which the system downtime is minimized, which has a direct correlation to the number of capability exchanges required to deliver the new configuration. Thus the sequence is identified by finding the production sequence which has i) the highest total similarity value and ii) a feasible sequence delivering all of the products. In this example the four highest value relationships are: $\mathrm{BE}, \mathrm{CE}, \mathrm{AD}$ and AC. This results in two possible sequences:

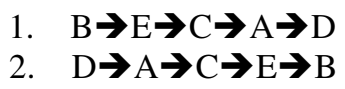

As one is the reverse of the other, the decision as to which one to choose is based upon the similarity of the two products at the ends of the sequence to the existing system. The result of this is that the final production sequence is:

$\mathrm{B} \rightarrow \mathrm{E} \rightarrow \mathrm{C} \rightarrow \mathrm{A} \rightarrow \mathrm{D}$.

\section{Conclusions and Further Work}

This paper has presented a methodology for the planning of multiple reconfigurations of a reconfigurable assembly system. This methodology has been demonstrated through a simple example and shown to aid in the identification of capabilities to remove, retain and procure. Furthermore it has demonstrated that the optimal production sequence can be identified. 
The next stages of work will address the finer details of the Reconfiguration Methodology and it's application to software. It will also investigate the equipment allocation, which will include developing a library of equipment modules. Furthermore, more detailed test cases based upon commercially available/destined products will be developed to apply to and assess the methodology.

\section{Acknowledgments}

The authors would like to acknowledge the Engineering and Physical Sciences Research Council (EPSRC) in the United Kingdom for the funding which has enabled this research to be conducted.

\section{References}

1. Koren, Y.: Report to Industry. The NSF Engineering Research Center for Reconfigurable Manufacturing Systems, University of Michigan College of Engineering (2004)

2. Du, S., Xi, L., Ni, J., Ershun, P., Liu, C.R.: Product lifecycle-oriented quality and productivity improvement based on stream of variation methodology. Computers in Industry 59(2-3), 180-192 (2008)

3. Vos, J.A.W.M.: Module and System Design in Flexibly Automated Assembly, TU Delft. $\mathrm{PhD}(2001)$

4. Lohse, N.: Towards an Ontology Framework for the Integrated Design of Modular Assembly Systems, The University of Nottingham. PhD (2006)

5. De Lit, P., Delchambre, A.: Integrated Design of a Product Family and its Assembly System. Kluwer Academic Publishers Group, The Netherlands (2003)

6. Sujono, S., Lashkari, R.S.: A multi-objective model of operation allocation and material handling system selection in FMS design. International Journal of Production Economics 105(1), 116-133 (2007)

7. Smale, D., Ratchev, S.: A Capability Model and Taxonomy for Multiple Assembly System Reconfigurations. In: 13th IFAC Symposium on Information Control Problems in Manufacturing, Moscow, Russia, June 3-5. Elsevier, Amsterdam (2009)

8. Smale, D., Ratchev, S.: Enabling a Reconfiguration Methodology for Multiple Assembly System Reconfigurations. In: COMEH (Consortium of UK University Manufacturing and Engineering) ICMR International Conference on Manufacturing Research, Warwick, United Kingdom, September 8-10 (2009)

9. Smale, D., Ratchev, S.: A Reconfiguration Methodology for Multiple Assembly System Reconfigurations. In: ASME (American Society of Mechanical Engineers) 4th International Manufacturing Science and Engineering Conference, West Lafayette, IN, USA, October 4-7 (2009) 